\title{
On-Line Choice Number of Complete Multipartite Graphs: an Algorithmic Approach
}

\author{
Fei-Huang Chang* \\ Division of Preparatory Programs \\ for Overseas Chinese Students \\ National Taiwan Normal University \\ New Taipei, Taiwan \\ cfh@ntnu.edu.tw \\ Jun-Yi Guo ${ }^{\dagger}$ \\ Department of Mathematics \\ National Taiwan Normal University \\ Taipei, Taiwan \\ junyiguo@gmail.com
}

\author{
Hong-Bin Chen
}

Institute of Mathematics

Academia Sinica

Taipei, Taiwan

hbchen@math.sinica.edu.tw

Yu-Pei Huang $\ddagger$

Department of Applied Mathematics

I-Shou University

Kaohsiung, Taiwan

yphuang@isu.edu.tw

Submitted: May 13, 2013; Accepted: Dec 19, 2014; Published: Jan 2, 2015

Mathematics Subject Classifications: 05C15, 05C57, 05C85

\begin{abstract}
This paper studies the on-line choice number of complete multipartite graphs with independence number $m$. We give a unified strategy for every prescribed $m$. Our main result leads to several interesting consequences comparable to known results. (1) If $k_{1}-\sum_{p=2}^{m}\left(\frac{p^{2}}{2}-\frac{3 p}{2}+1\right) k_{p} \geqslant 0$, where $k_{p}$ denotes the number of parts of cardinality $p$, then $G$ is on-line chromatic-choosable. (2) If $|V(G)| \leqslant \frac{m^{2}-m+2}{m^{2}-3 m+4} \chi(G)$, then $G$ is on-line chromatic-choosable. (3) The on-line choice number of regular complete multipartite graphs $K_{m \star k}$ is at most $\left(m+\frac{1}{2}-\sqrt{2 m-2}\right) k$ for $m \geqslant 3$.
\end{abstract}

Keywords: on-line list coloring; Ohba's conjecture

\section{Introduction}

On-line list coloring was introduced by Schauz [13] in the context of the Paint-Correct game played on a graph. Here we adapt the on-line list coloring version introduced by

*ROC National Science Council grant NSC101-2115-M-003-007.

${ }^{\dagger}$ ROC Ministry of Science and Technology grant 103-2511-S-003-006-.

${ }^{\ddagger}$ ROC Ministry of Science and Technology grant 103-2811-M-214-001 and 103-2632-M-214-001-MY3-1. 
Zhu [15] as follows.

Definition 1. Given a graph $G$ and an integer-valued function $f$ on $V(G)$, the on-line $f$-list coloring of $G$ is a two-players game, say Alice and Bob, played on $G$. In the very beginning, all vertices are uncolored. In the $i$ th round, Alice marks a nonempty subset $V_{i}$ of remaining uncolored vertices and assigns color $i$ as a permissible color to each vertex of $V_{i}$. Then Bob chooses an independent set $X_{i}$ contained in $V_{i}$ and colors all vertices of $X_{i}$ the color $i$. The game goes round by round. If at the end of some round there is a vertex $v$ which has been assigned $f(v)$ permissible colors, i.e., has been marked $f(v)$ times, but is not yet colored by Bob, then Alice wins the game. Otherwise, Bob wins, i.e., in the end each vertex $v$ is colored by Bob before running out of $f(v)$ permissible colors.

Given an integer-valued function $f$ defined on $V(G)$, we say that $G$ is on-line $f$ choosable if Bob has a winning strategy for the on-line $f$-list coloring game on $G$ no matter how Alice plays; particularly, if $f(v)$ is a constant $k$ for all $v \in V(G)$, then we say that $G$ is on-line $k$-choosable. Denoted by $\chi_{p}(G)$, the on-line choice number of $G$ is the minimum number $k$ such that $G$ is on-line $k$-choosable.

The conventional list coloring, introduced by Vizing [14] and independently by Erdös, Rubin and Taylor [3], is a special case that Alice shows Bob the full lists in the very beginning of the on-line list coloring game. So Bob has a winning strategy for the list coloring if he has one for the on-line list coloring game. Let $\chi(G)$ and $\chi_{\ell}(G)$ denote the chromatic number and choice number of a graph $G$, respectively. In general, we have $\chi(G) \leqslant \chi_{\ell}(G) \leqslant \chi_{p}(G)$ for any $G$.

It is known that $\chi_{\ell}(G)-\chi(G)$ can be arbitrarily large; see [5] for an example that demonstrates complete bipartite graphs $G$ having $\chi_{\ell}(G)$ arbitrarily large but $\chi(G)=2$. An interesting question is whether $\chi_{p}(G)-\chi_{\ell}(G)$ can be arbitrarily large. To the best of our knowledge, the problem is still open. Although there exist a few graphs $G$ with $\chi_{p}(G)>\chi_{\ell}(G)$, the largest gap known up to now is 1 as shown in [15].

Another interesting question raises naturally: for which graphs $G$ does $\chi(G)=\chi_{\ell}(G)$ $=\chi_{p}(G)$ ? There have been many studies on graphs satisfying $\chi_{\ell}(G)=\chi(G)$ (see $[4,7$, $9,12]$ and references therein); such a graph is called chromatic-choosable. Likewise, a graph $G$ is called on-line chromatic-choosable if $\chi_{p}(G)=\chi(G)$. Ohba [12] conjectured that for all graphs $G$ with $|V(G)| \leqslant 2 \chi(G)+1, G$ is chromatic-choosable; recently, this has been proved by Noel, Reed and Wu [11]. Let $K_{n_{1} \star k_{1}, n_{2} \star k_{2}, \ldots, n_{s} \star k_{s}}$ denote the complete multipartite graph where $k_{i}$ partite sets are of size $n_{i}$ for $i=1,2, \ldots, s$. For short, we shall simplify $n_{i} \star 1$ as $n_{i}$ (for example $K_{3 \star 2,4}=K_{3 \star 2,4 \star 1}$ ). In view of the fact that $K_{2 \star k, 3}$ is not on-line chromatic-choosable for $k \geqslant 2$ [8], Huang, Wong and Zhu [6] slightly modified the Ohba's conjecture to its on-line version.

Conjecture 2. [6] Every graph $G$ with $|V(G)| \leqslant 2 \chi(G)$ is on-line chromaticchoosable.

We remark that to prove the on-line Ohba's conjecture, it suffices to prove it for complete $\chi(G)$-partite graphs $G$ since adding edges does not reduce the on-line choice number. Conjecture 2 has been verified for complete multipartite graphs with a small 
independence number. Using the Combinatorial Nullstellensatz, the authors [6] proved that $K_{2 \star k}$ is on-line chromatic-choosable. Recently, Kim et al [8] gave an algorithmic proof for $K_{2 \star k}$ and later Kozik, Micek and Zhu [10] extended the case to complete multipartite graphs with independence number at most 3.

This paper focuses on complete multipartite graphs with independence number $m$. In Section 2, we generalize the algorithmic methods in $[8,10]$ and give a unified strategy for the on-line choice number of graphs with any prescribed $m$. Our main result provides a sufficient condition on $f$ for graphs being on-line $f$-choosable by partitioning vertices into independent sets in a systematic way. It is a broadly applicable tool which leads to several interesting consequences comparable to known results. Section 3 presents some immediate consequences.

\section{Main Result}

This section starts with some notations and definitions. Throughout the rest of this paper, we shall use "part" instead of "partite set" for short, and let $m$ be a fixed positive integer. Consider a complete multipartite graph $G$ with part size at most $m$, i.e. independent number at most $m$. Let $\Pi=\left\{\underline{X}_{m-1}, \underline{X}_{m-2}, \ldots, \underline{X}_{1}, \bar{X}_{2}, \ldots, \bar{X}_{m}\right\}$ be a partition of parts of $G$, where $\bar{X}_{p}$ is a family consisting of parts of size exact $p$ for $2 \leqslant p \leqslant m$ and $\underline{X}_{p}$ a family consisting of parts of size at most $p$ for $1 \leqslant p \leqslant m-1$. Particularly, $\underline{X}_{1}$ contains only parts of size 1 . Notably, the partition is not unique since a part of size $p$ can belong to either $\bar{X}_{p}$ or $\underline{X}_{j}$ for some $j \leqslant p$. Let $u_{p}$ and $\ell_{p}$ denote the number of parts of $\bar{X}_{p}$ and $\underline{X}_{p}$, respectively, i.e., $\left|\bar{X}_{p}\right|=u_{p}$ and $\left|\underline{X}_{p}\right|=\ell_{p}$. For each family $\underline{X}_{p}$, we use the second coordinates to denote parts in the family, e.g., $\underline{X}_{p}=\left(\underline{X}_{p, 1}, \ldots, \underline{X}_{p, \ell_{p}}\right)$ where $\underline{X}_{p, i}$ means the $i$ th part in $\underline{X}_{p}$. When it comes to vertices in a family of parts, we shall use the notation $V(\cdot)$ to avoid confusion. Given a function $f: V(G) \rightarrow \mathbb{N}$, for $y=1,2, \ldots, m$, define

$$
F(y) \equiv \min \left\{\sum_{v \in V(Y)} f(v): Y \subseteq X \in \bigcup_{p=2}^{m} \bar{X}_{p} \text { and }|Y|=y\right\} .
$$

Notice that $F(1)=\min _{v \in \bigcup_{p=2}^{m} V\left(\bar{X}_{p}\right)} f(v)$. For $j=1,2, \ldots, m$, define

$$
S(j) \equiv \sum_{p=2}^{j} u_{p}+\sum_{p=j+1}^{m}(p-j) u_{p}
$$

Let $\alpha(1)=\sum_{p=2}^{m} u_{p}$ and $\beta(1)=0$, and define recursively that for $j=2, \ldots, m$,

$$
\begin{aligned}
& \alpha(j) \equiv \alpha(j-1)+S(j-1) \text { and } \\
& \beta(j) \equiv \beta(j-1)+\sum_{p=1}^{m-j+1}\left|V\left(\underline{X}_{p}\right)\right| .
\end{aligned}
$$


Then it can be easily expressed as

$$
\alpha(j)=\sum_{p=2}^{j}\left(j+\frac{p^{2}}{2}-\frac{3 p}{2}+1\right) u_{p}+\sum_{p=j+1}^{m}\left(\frac{j}{2}-\frac{j^{2}}{2}+p j-p+1\right) u_{p}
$$

and

$$
\beta(j)=\sum_{p=1}^{m-j}(j-1)\left|V\left(\underline{X}_{p}\right)\right|+\sum_{p=m-j+1}^{m-1}(m-p)\left|V\left(\underline{X}_{p}\right)\right|
$$

for $j=1, \ldots, m$.

The following propositions are elementary but useful observations.

Proposition 3. For $j=1, \ldots, m-1$, let $S(m-j)=\sum_{p=2}^{m} s_{p} u_{p}$. Then we have $1=s_{2}=$ $s_{3}=\cdots=s_{m-j+1}<s_{m-j+2}<\cdots<s_{m-1}<s_{m}$.

Proof. The proof follows immediately by definition.

Proposition 4. For $j \geqslant 2$, let $\alpha(j)+\beta(j)=\sum_{p=2}^{m} a_{p} u_{p}+\sum_{p=1}^{m-1} b_{p}\left|V\left(\underline{X}_{p}\right)\right|$ and $a_{1}=b_{1}$. The following is true.

(i) $b_{1}=b_{2}=\cdots=b_{m-j+1}=j-1$.

(ii) For any integers $s$ and $t$ with $1 \leqslant s<t \leqslant m$, we have $a_{t}-a_{s} \geqslant t-s$. In particular, if $t>j$, then $a_{t}-a_{s} \geqslant j-1$ and if $t \leqslant j$, then $a_{t}-a_{s} \geqslant t-2$.

(iii) For $p=2,3, \ldots, m$, we have $a_{p} \geqslant \max \{j, p\}$.

Proof. The proof of (i) is trivial. We first prove (ii) and consider the following cases.

$s=1 \quad$ (a) If $2 \leqslant j<t \leqslant m$, then $\left(a_{t}-a_{s}\right)-(t-s)=\left(a_{t}-a_{1}\right)-(t-1)$

$$
\begin{aligned}
& =\left(\left(\frac{j}{2}-\frac{j^{2}}{2}+t j-t+1\right)-(j-1)\right)-(t-1) \\
& =\frac{j}{2}-\frac{j^{2}}{2}+t(j-2)+3 \geqslant \frac{j}{2}-\frac{j^{2}}{2}+(j+1)(j-2)+3 \\
& =\frac{j(j-1)+2}{2} \geqslant 0 . \text { Thus, } a_{t}-a_{s} \geqslant \max \{t-s, j-1\} .
\end{aligned}
$$

(b) If $1<t \leqslant j \leqslant m$, then $\left(a_{t}-a_{s}\right)-(t-s)=\left(a_{t}-a_{1}\right)-(t-1)=((j+$ $\left.\left.\frac{t^{2}}{2}-\frac{3 t}{2}+1\right)-(j-1)\right)-(t-1)=\frac{1}{2}(t-2)(t-3) \geqslant 0($ as $t \in \mathbb{N})$. Therefore, $a_{t}-a_{s} \geqslant \max \{t-s, t-2\}$.

$s>1$ (a) If $2 \leqslant j<s<t \leqslant m$, then we have $a_{t}-a_{s}=\left(\frac{j}{2}-\frac{j^{2}}{2}+t j-t+1\right)-\left(\frac{j}{2}-\frac{j^{2}}{2}+\right.$ $s j-s+1)=(t-s)(j-1) \geqslant \max \{t-s, j-1\}$. 
(b) If $2 \leqslant s \leqslant j<t \leqslant m$, then

$$
\begin{aligned}
\left(a_{t}-a_{s}\right)-(t-s) & =\left(\left(\frac{j}{2}-\frac{j^{2}}{2}+t j-t+1\right)-\left(j+\frac{s^{2}}{2}-\frac{3 s}{2}+1\right)\right)-(t-s) \\
& =\frac{1}{2}\left(2 t(j-2)-j-j^{2}-s^{2}+5 s\right) \\
& \geqslant \frac{1}{2}\left(2(j+1)(j-2)-j-j^{2}-s^{2}+5 s\right) \\
& =\frac{1}{2}((j+s-3)(j-s)+2(s-2)) \geqslant 0 .
\end{aligned}
$$

Besides,

$$
\begin{aligned}
\left(a_{t}-a_{s}\right)-(j-1) & =\left(\left(\frac{j}{2}-\frac{j^{2}}{2}+t j-t+1\right)-\left(j+\frac{s^{2}}{2}-\frac{3 s}{2}+1\right)\right)-(j-1) \\
& =\frac{1}{2}\left(2 t(j-1)-j^{2}-3 j+2-\left(s^{2}-3 s\right)\right) \\
& \geqslant \frac{1}{2}\left(2(j+1)(j-1)-j^{2}-3 j+2-\left(s^{2}-3 s\right)\right) \\
& =\frac{1}{2}\left(\left(j^{2}-3 j\right)-\left(s^{2}-3 s\right)\right) \geqslant 0,
\end{aligned}
$$

where the last inequality holds for $j \geqslant s \geqslant 2$. Therefore, $a_{t}-a_{s} \geqslant \max \{t-$ $s, j-1\}$.

(c) If $2 \leqslant s<t \leqslant j \leqslant m$, then $a_{t}-a_{s}=\left(j+\frac{t^{2}}{2}-\frac{3 t}{2}+1\right)-\left(j+\frac{s^{2}}{2}-\frac{3 s}{2}+1\right)=$ $\frac{1}{2}(t-s)(t+s-3)$. If $s=t-1$, then $a_{t}-a_{s}=t-2 \geqslant t-s$. If $s<t-1$, then $t-s \geqslant 2$ and $a_{t}-a_{s} \geqslant t+s-3 \geqslant t-1>t-2 \geqslant t-s$. In either case, $a_{t}-a_{s} \geqslant \max \{t-s, t-2\}$, as desired.

Next, we prove (iii). When $p \leqslant j$, we have $j+\frac{p^{2}}{2}-\frac{3 p}{2}+1-j=\frac{p^{2}-3 p+2}{2} \geqslant 0$ for all $p \geqslant 2$. When $p \geqslant j+1$,

$$
\begin{aligned}
\frac{j}{2}-\frac{j^{2}}{2}+p j-p+1-p & =\frac{j}{2}-\frac{j^{2}}{2}+(j-2) p+1 \\
& \geqslant \frac{j}{2}-\frac{j^{2}}{2}+(j-2)(j+1)+1 \\
& =\frac{1}{2}\left(j^{2}-j-2\right) \geqslant 0
\end{aligned}
$$

for all $j \geqslant 2$. The proof is complete.

Throughout the paper, $U$ shall be used to denote the set Alice marks and $I \subseteq U$ denotes the set Bob removes. For any $U \subseteq V(G)$, the indicator function $\mathbf{1}_{U}$ of $U$ is defined as $\mathbf{1}_{U}(x)= \begin{cases}1 & \text { if } x \in U ; \\ 0 & \text { if } x \notin U\end{cases}$

Proposition 5. [10, 13$]$ If $G$ is edgeless and $f(v) \geqslant 1$ for all $v \in V(G)$, then $G$ is on-line $f$-choosable. A graph $G$ is on-line $f$-choosable if and only if for any $U \subseteq V(G)$ there exists an independent set $I \subseteq U$ of $G$ such that $G-I$ is on-line $\left(f-\mathbf{1}_{U}\right)$-choosable.

For a subset $Y \subseteq X \in \bar{X}_{j}$ with $|Y|=y$, we say that $F(y)$ is saturated with respect to $Y$ if $F(y)=\sum_{w \in Y} f(w)=\alpha(j)+\beta(j)$. We are now ready to prove the main result.

Theorem 6. Let $G$ be a complete multipartite graph with independence number $m \geqslant 2$. If there is a partition $\Pi$ of parts of $G$ and a function $f: V(G) \rightarrow \mathbb{N}$ satisfying the following: 
(R1)

$F(j) \geqslant \alpha(j)+\beta(j)$ for all $j=1, \ldots, m$ and

(R2) $f(v) \geqslant S(m-j)+\sum_{p=1}^{j-1}\left|V\left(\underline{X}_{p}\right)\right|+\sum_{q=1}^{i-1}\left|V\left(\underline{X}_{j, q}\right)\right|+1$ for all $v \in V\left(\underline{X}_{j, i}\right)$ for all $j=1, \ldots, m$ and $1 \leqslant i \leqslant \ell_{j}$,

then $G$ is on-line $f$-choosable.

Proof. We shall prove the theorem by induction on $|V(G)|$. Obviously, if $G$ is edgeless, then $G$ is on-line $f$-choosable since $f(v) \geqslant F(1) \geqslant 1$ for all $v \in V\left(\bigcup_{p=2}^{m} \bar{X}_{p}\right)$ by (R1) and $f(v) \geqslant 1$ for $v \in V\left(\bigcup_{p=1}^{m-1} \underline{X}_{p}\right)$ by (R2). Assume that $G$ has at least two parts and that the statement is true for all graphs of order less than $|V(G)|$. We shall prove that if $G$ has a partition $\Pi$ of parts and a function $f: V(G) \rightarrow \mathbb{N}$ so that (R1) and (R2) are satisfied, then no matter what $U \subseteq V(G)$ Alice marks, there exists an independent set $I \subseteq U$ of $G$ such that the resulting graph $G^{\prime}=G-I$ satisfies the two conditions, i.e., there exists a partition $\Pi^{\prime}$ of parts of $G^{\prime}$ such that $f^{\prime}=f-\mathbf{1}_{U}$ satisfies (R1) and (R2) with respect to $\Pi^{\prime}$. Then by induction we conclude that $G^{\prime}$ is on-line $f^{\prime}$-choosable and thus $G$ is on-line $f$-choosable by Proposition 5 .

For a given $U \subseteq V(G)$, the crucial step is twofold: decide an independent set $I \subseteq U$ and give a partition $\Pi^{\prime}=\left\{\underline{X}_{m-1}^{\prime}, \underline{X}_{m-2}^{\prime}, \ldots, \underline{X}_{1}^{\prime}, \bar{X}_{2}^{\prime}, \ldots, \bar{X}_{m}^{\prime}\right\}$ of parts of $G^{\prime}$. Our strategy will be given case by case depending on $U$. Particularly, in any considered case we shall assume that all the previous cases do not hold. Note that from $\Pi$ to $\Pi^{\prime}$ all families are inherited except two: the family from which $I$ is chosen and the family where the remaining partite set $X-I$ is inserted. The notations $\left(\underline{X}_{j}, X-I\right)$ and $\left(X-I, \underline{X}_{j}\right)$ denote that the remaining set $X-I$ is inserted to the end and the beginning of the family $\underline{X}_{j}$, respectively. Note also that, once $U$ is given, the function $f^{\prime}$ can be obtained from $f$ with little difference $\mathbf{1}_{U}$. So we may and shall verify the inequalities in (R1) and (R2) for $f^{\prime}$ and $\Pi^{\prime}$ by comparing the difference with that for $f$ and $\Pi$.

Case 1: $U$ contains a part $X \in \bar{X}_{j^{*}}$ for some $j^{*}$.

Let $I=X$ and $\Pi^{\prime}$ be obtained from $\Pi$ by removing $I$ where all families remain except $\bar{X}_{j^{*}}^{\prime}=\bar{X}_{j^{*}}-X$. Next we verify (R1) and (R2) for the updated $\Pi^{\prime}$ and $f^{\prime}$.

(R1). For all $j \in[m]$, we have $F^{\prime}(j) \geqslant F(j)-j \geqslant \alpha(j)+\beta(j)-j \geqslant \alpha^{\prime}(j)+\beta^{\prime}(j)$, where the last inequality follows from $u_{j^{*}}^{\prime}=u_{j^{*}}-1, \beta^{\prime}(j)=\beta(j)$ and Proposition 4.

(R2). Since $f^{\prime}(v) \geqslant f(v)-1$ for each $v \in V\left(\underline{X}_{j}\right)$, it suffices to show that $S^{\prime}(m-j) \leqslant$ $S(m-j)-1$. This follows immediately from $u_{j^{*}}^{\prime}=u_{j^{*}}-1$ and Proposition 3.

Case 2: $U \cap X \neq \emptyset$ for some $X \in \bar{X}_{j^{*}}$ and $F(y)$ is saturated with respect to $\boldsymbol{Y}$ for some $\boldsymbol{Y} \subseteq U \cap X$, where $\boldsymbol{y}=|\boldsymbol{Y}|$.

Among all those cases we choose the one with the largest $y$. Let $I=U \cap X$ and $\Pi^{\prime}$ be obtained from $\Pi$ by removing $I$ where all families remain except $\bar{X}_{j^{*}}^{\prime}=\bar{X}_{j^{*}}-X$ and $\underline{X}_{m-y}^{\prime}=\left(\underline{X}_{m-y}, X-I\right)$. Let $y^{*}=|U \cap X|$. Obviously, $u_{j^{*}}^{\prime}=u_{j^{*}}-1, \ell_{m-y}^{\prime}=\ell_{m-y}+1$ and $\left|V\left(\underline{X}_{m-y}^{\prime}\right)\right|=\left|V\left(\underline{X}_{m-y}\right)\right|+\left(j^{*}-y^{*}\right)$. Of particular note is that $j^{*}>y$ otherwise it is Case 1. 
(R1). Consider $F^{\prime}(j)$ for the case $j \leqslant y$, which implies $j^{*}>j$. Since $j^{*}>j$ and $u_{j^{*}}^{\prime}=u_{j^{*}}-1$, from Eq.(1) we obtain

$$
\alpha(j)=\alpha^{\prime}(j)+\left(\frac{j}{2}-\frac{j^{2}}{2}+j j^{*}-j^{*}+1\right) .
$$

Since there are $j^{*}-y^{*}$ elements inserted to the family $\underline{X}_{m-y}^{\prime}$ and $m-y \leqslant m-j$, by Proposition $4, \beta(j)=\beta^{\prime}(j)-(j-1)\left(j^{*}-y^{*}\right)$. We now verify $(\mathrm{R} 1)$,

$$
\begin{aligned}
\mathrm{F}^{\prime}(j) & \geqslant F(j)-j \\
& \geqslant \alpha(j)+\beta(j)-j \\
& =\left[\alpha^{\prime}(j)+\left(\frac{j}{2}-\frac{j^{2}}{2}+j j^{*}-j^{*}+1\right)\right]+\left[\beta^{\prime}(j)-(j-1)\left(j^{*}-y^{*}\right)\right]-j \\
& =\alpha^{\prime}(j)+\beta^{\prime}(j)+(j-1)\left(y^{*}-\frac{j+2}{2}\right) \\
& \geqslant \alpha^{\prime}(j)+\beta^{\prime}(j) \quad\left(\text { as } y^{*} \geqslant y \geqslant j\right) .
\end{aligned}
$$

Consider the case $j>y$. Since $j^{*}>y$, it follows that $m-y \geqslant m-j^{*}+1$ and the coefficient of $\left|V\left(\underline{X}_{m-y}\right)\right|$ in the expression (2) of $\beta(j)$ is $y$. Thus, $\beta(j)=\beta^{\prime}(j)-y\left(j^{*}-y^{*}\right)$. We have $F^{\prime}(j) \geqslant F(j)-j \geqslant \underbrace{\alpha(j)+\beta(j)-j+1}_{Q}$ where the second inequality holds by the maximality assumption of $y$.

If $j<j^{*}$, then

$$
\begin{aligned}
Q & =\underbrace{\alpha^{\prime}(j)+\left(\frac{j}{2}-\frac{j^{2}}{2}+j j^{*}-j^{*}+1\right)}_{\text {by Eq. }(3)}+\beta^{\prime}(j)-y\left(j^{*}-y^{*}\right)-j+1 \\
& \geqslant \alpha^{\prime}(j)+\left(\frac{j}{2}-\frac{j^{2}}{2}+j j^{*}-j^{*}+1\right)+\beta^{\prime}(j)-y^{*}\left(j^{*}-y^{*}\right)-j+1 \\
& \geqslant \alpha^{\prime}(j)+\beta^{\prime}(j),
\end{aligned}
$$

where the last inequality can be proved through two cases: If $y^{*} \geqslant j$, then it follows from the same analysis as the previous case; if $y^{*}<j$, then it follows from the fact that the quadratic function $g(j)=\left(\frac{j}{2}-\frac{j^{2}}{2}+j j^{*}-j^{*}+1\right)-y^{*}\left(j^{*}-y^{*}\right)-j+1$ is increasing when $y^{*}<j<j^{*}$ and $g\left(y^{*}+1\right) \geqslant 0$ for $y^{*} \notin(1,2)$.

If $j \geqslant j^{*}$, then the coefficient of $u_{j^{*}}$ in the expression (1) of $\alpha(j)$ is $j+\frac{\left(j^{*}\right)^{2}}{2}-\frac{3 j^{*}}{2}+1$, which implies $\alpha(j)=\alpha^{\prime}(j)+\left(j+\frac{\left(j^{*}\right)^{2}}{2}-\frac{3 j^{*}}{2}+1\right)$. Hence

$$
\begin{aligned}
Q & =\left[\alpha^{\prime}(j)+\left(j+\frac{\left(j^{*}\right)^{2}}{2}-\frac{3 j^{*}}{2}+1\right)\right]+\beta^{\prime}(j)-y\left(j^{*}-y^{*}\right)-j+1 \\
& \geqslant \alpha^{\prime}(j)+\beta^{\prime}(j)+\frac{\left(j^{*}\right)^{2}}{2}-\frac{3 j^{*}}{2}+2-y\left(j^{*}-y\right)
\end{aligned}
$$




$$
\begin{aligned}
& \geqslant \alpha^{\prime}(j)+\beta^{\prime}(j)+\frac{\left(j^{*}\right)^{2}}{2}-\frac{3 j^{*}}{2}+2-\frac{\left(j^{*}\right)^{2}}{4} \quad\left(\text { as } y\left(j^{*}-y\right) \text { has a max. at } y=\frac{j^{*}}{2}\right) \\
& =\alpha^{\prime}(j)+\beta^{\prime}(j)+\frac{\left(j^{*}-2\right)\left(j^{*}-4\right)}{4} \\
& \left.\geqslant \alpha^{\prime}(j)+\beta^{\prime}(j) \text { whenever } j^{*} \neq 3 \text { (noticing that } j^{*} \geqslant 2\right) .
\end{aligned}
$$

When $j^{*}=3$, either $y=1$ or $y=2$. This implies $y\left(j^{*}-y\right)=2$. Consequently, $\frac{\left(j^{*}\right)^{2}}{2}-\frac{3 j^{*}}{2}+2-y\left(j^{*}-y\right)=0$ and then $F^{\prime}(j) \geqslant Q \geqslant \alpha^{\prime}(j)+\beta^{\prime}(j)$, as desired.

(R2). Consider $f^{\prime}(v)$ for any $v \in \underline{X}_{j, i}^{\prime}$.

For the case $\{j<m-y\}$ or $\left\{j=m-y\right.$ and $\left.i \leqslant \ell_{m-y}\right\}$, we conclude the same

$$
\begin{aligned}
f^{\prime}(v) \geqslant f(v)-1 & \geqslant S(m-j)+\sum_{p=1}^{j-1}\left|V\left(\underline{X}_{p}\right)\right|+\sum_{q=1}^{i-1}\left|V\left(\underline{X}_{j, q}\right)\right| \\
& \geqslant S^{\prime}(m-j)+1+\sum_{p=1}^{j-1}\left|V\left(\underline{X}_{p}^{\prime}\right)\right|+\sum_{q=1}^{i-1}\left|V\left(\underline{X}_{j, q}^{\prime}\right)\right|,
\end{aligned}
$$

where the last inequality follows from $u_{j^{*}}^{\prime}=u_{j^{*}}-1$, Proposition 3 and the fact that the last two terms are inherited.

For the case $j=m-y$ and $i=\ell_{m-y}+1$, obviously $v \in X-I$. In this case,

$$
\begin{aligned}
f^{\prime}(v)=f(v) & \geqslant F(y+1)-\sum_{w \in Y} f(w) \quad(\text { as } F(y) \text { is saturated with respect to } Y) \\
& =F(y+1)-[\alpha(y)+\beta(y)] \\
& \geqslant \alpha(y+1)-\alpha(y)+\beta(y+1)-\beta(y) \\
& =S(y)+\sum_{p=1}^{m-y}\left|V\left(\underline{X}_{p}\right)\right| \\
& \left.\geqslant\left[S^{\prime}(y)+1\right]+\left[\sum_{p=1}^{m-y-1}\left|V\left(\underline{X}_{p}^{\prime}\right)\right|+\sum_{q=1}^{i-1} \mid V\left(\underline{X}_{m-y, q}\right)\right]\right] \text { (by Proposition 3) }
\end{aligned}
$$

and thus (R2) is satisfied by simply substituting $m-j$ into $y$.

For the case $j>m-y$, we have $j^{*}>y^{*} \geqslant y \geqslant m-j+1$. Therefore, by definition, the coefficient of $u_{j^{*}}$ in the expression of $S(m-j)$ is $j^{*}-m+j$. This implies $S^{\prime}(m-j) \leqslant$ $S(m-j)-\left(j^{*}-m+j\right)$ and thus

$$
\begin{aligned}
f^{\prime}(v) & \geqslant f(v)-1 \\
& \geqslant S(m-j)+\left[\sum_{p=1}^{j-1}\left|V\left(\underline{X}_{p}\right)\right|+\sum_{q=1}^{i-1} \mid V\left(\underline{X}_{j, q}\right)\right] \\
& \geqslant S^{\prime}(m-j)+\left(j^{*}-m+j\right)+\left[\sum_{p=1}^{j-1}\left|V\left(\underline{X}_{p}^{\prime}\right)\right|+\sum_{q=1}^{i-1}\left|V\left(\underline{X}_{j, q}^{\prime}\right)\right|-\left(j^{*}-y^{*}\right)\right]
\end{aligned}
$$




$$
\geqslant S^{\prime}(m-j)+\sum_{p=1}^{j-1}\left|V\left(\underline{X}_{p}^{\prime}\right)\right|+\sum_{q=1}^{i-1}\left|V\left(\underline{X}_{j, q}^{\prime}\right)\right|+1
$$

as desired.

Note that as Cases 1 and 2 were excluded, in all remaining cases we conclude that if $U \cap X \neq \emptyset$ for some $X \in \bar{X}_{j}$, then

(i) $|U \cap X|<j$ otherwise it is Case 1,

(ii) for all subsets $Y \subseteq U \cap X, F(|Y|)$ is not saturated with respect to $Y$ otherwise it is Case 2.

Case 3: From the above discussion, we know that one of the following cases must happen if $U \neq \emptyset$.

$V\left(\underline{X}_{m+1-s}\right) \cap U \neq \emptyset$ for some $2 \leqslant s \leqslant m$,

(3.2) there exists $Y \subseteq U$ and $Y \subseteq X \in \bigcup_{p=2}^{m} \bar{X}_{p}$ with $|Y|=t$ for some $1 \leqslant t \leqslant$ $m-1$ such that $F(t)$ is not saturated with respect to $Y$,

(3.3) there exists $Y \nsubseteq U$ and $Y \subseteq X \in \bar{X}_{u}$ for some $2 \leqslant u \leqslant m$ such that $F(|Y|)$ is saturated with respect to $Y$ and $X \cap U \neq \emptyset$.

Among all these cases, we choose the one with the largest $s, t, u$ (if they are equal then the priority is $s, t$ and then $u$ ). For example, if $s=u=4$ and $t=5$ then the first case we deal with is Case (3.2) with $t=5$.

Case (3.1): Notice that in this case, $s \geqslant \boldsymbol{u}$ and $\boldsymbol{s} \geqslant \boldsymbol{t}$. We choose the least $i^{*}$ among all $i$ 's with $V\left(\underline{X}_{m+1-s, i}\right) \cap U \neq \emptyset$. Let $I=V\left(\underline{X}_{m+1-s, i^{*}}\right) \cap U$ and $\Pi^{\prime}$ be obtained from $\Pi$ by removing $I$ where all families remain except $\underline{X}_{m+1-s, i^{*}}^{\prime}=\underline{X}_{m+1-s, i^{*}}-I$ and the orderings are inherited. Obviously, $\alpha^{\prime}(j)=\alpha(j)$ for all $j \in[m]$.

(R1). Consider any subset $J \subseteq X \in \bar{X}_{j^{*}}$ with $|J|=j$ for some $j^{*}$. Notice that $j \leqslant j^{*}$. Here we may assume that $J \cap U \neq \emptyset$ because it is trivial that $\sum_{w \in J} f^{\prime}(w) \geqslant \alpha^{\prime}(j)+\beta^{\prime}(j)$ if $J \cap U=\emptyset$.

If $F(j)$ is saturated with respect to $J$, then $J \nsubseteq U$ (by Case 2) and $j^{*} \leqslant s$ (otherwise it is Case (3.3) with $u=j^{*}>s$ ). Since $\underline{X}_{m+1-s}^{\prime}=\underline{X}_{m+1-s}-I, I \neq \emptyset$, and the coefficient of $\left|V\left(\underline{X}_{m+1-s}\right)\right|$ in the expression of $\beta(j)$ is $j-1$ (from $j \leqslant s$ and Proposition 4 ), we have $\beta(j) \geqslant \beta^{\prime}(j)+(j-1)$. It follows that

$$
\begin{aligned}
\sum_{w \in J} f^{\prime}(w) & \geqslant F(j)-(j-1) \quad(\text { as } J \nsubseteq U) \\
& \geqslant \alpha(j)+\beta(j)-(j-1) \\
& \geqslant \alpha^{\prime}(j)+\beta^{\prime}(j) . \quad\left(\text { as } \alpha^{\prime}(j)=\alpha(j)\right)
\end{aligned}
$$


If $F(j)$ is not saturated with respect to $J$ and $J \subseteq U$, then $j \leqslant s$ (otherwise it is Case (3.2) with $t=j>s)$. Thus, the coefficient of $\left|V\left(\underline{X}_{m+1-s}\right)\right|$ in $\beta(j)$ is $j-1$. This implies that $\beta(j) \geqslant \beta^{\prime}(j)+(j-1)$. Accordingly, $\sum_{w \in J} f^{\prime}(w) \geqslant[\alpha(j)+\beta(j)+1]-j \geqslant \alpha^{\prime}(j)+\beta^{\prime}(j)$, where the first inequality holds as $F(j)$ is not saturated with respect to $J$.

If $F(j)$ is not saturated and $J \nsubseteq U$, then $F(|J \cap U|)$ must also not be saturated with respect to $J \cap U$ (by Case 2) and $|J \cap U| \leqslant s$ (otherwise it is Case (3.2) with $t=|J \cap U|>s)$. Hence, $\sum_{w \in J} f^{\prime}(w) \geqslant F(j)+1-|J \cap U| \geqslant \alpha^{\prime}(j)+\beta^{\prime}(j)$. Notice that the last inequality holds as $\beta(j)+1-|J \cap U| \geqslant \beta^{\prime}(j)$, which follows from the fact that the coefficient of $\left|V\left(\underline{X}_{m+1-s}\right)\right|$ of $\beta(j)$ in Eq. $(2)$ is at least $\min \{j-1, s-1\}$.

From the above discussion, in either case we have $\sum_{w \in J} f^{\prime}(w) \geqslant \alpha^{\prime}(j)+\beta^{\prime}(j)$. As $J$ is chosen arbitrarily, we can conclude that $F^{\prime}(j) \geqslant \alpha^{\prime}(j)+\beta^{\prime}(j)$ for all $j \in[m]$.

(R2). Because of the maximality of $s$, it suffices to consider $v \in V\left(\underline{X}_{j, i}^{\prime}\right)$ for the two cases $\{j>m+1-s\}$ and $\left\{j=m+1-s\right.$ and $\left.i>i^{*}\right\}$ as $f^{\prime}(v)$ is inherited otherwise. If $j>m+1-s$, then $f^{\prime}(v) \geqslant f(v)-1 \geqslant S(m-j)+\sum_{p=1}^{j-1}\left|V\left(\underline{X}_{p}\right)\right|+\sum_{q=1}^{i-1}\left|V\left(\underline{X}_{j, q}\right)\right| \geqslant$ $S^{\prime}(m-j)+\left[\sum_{p=1}^{j-1}\left|V\left(\underline{X}_{p}^{\prime}\right)\right|+1\right]+\sum_{q=1}^{i-1}\left|V\left(\underline{X}_{j, q}^{\prime}\right)\right|$, as desired.

For the case that $i>i^{*}$ and $j=m+1-s$, because of the minimality of $i^{*}$, we have $\sum_{q=1}^{i-1}\left|V\left(\underline{X}_{j, q}\right)\right| \geqslant \sum_{q=1}^{i-1}\left|V\left(\underline{X}_{j, q}^{\prime}\right)\right|+1$. Similarly, we can conclude that $f^{\prime}(v) \geqslant$ $S^{\prime}(m-j)+\sum_{p=1}^{j-1}\left|V\left(\underline{X}_{p}^{\prime}\right)\right|+\left[\sum_{q=1}^{i-1}\left|V\left(\underline{X}_{j, q}^{\prime}\right)\right|+1\right]$, as desired.

Case (3.2): Note that in this case, $t$ is the largest, i.e., $t>s$ and $t \geqslant u$. We may assume that there exists $Y \subseteq U$ and $Y \subseteq X \in \bar{X}_{j^{*}}$ with $|Y|=t, 1 \leqslant t \leqslant m-1$, such that $F(t)$ is not saturated with respect to $Y$. Let $I=U \cap X$ (noticing that $j^{*}>|I| \geqslant t$ ) and $\Pi^{\prime}$ be obtained from $\Pi$ by removing $I$ where all families remain except that $\bar{X}_{j^{*}}^{\prime}=\bar{X}_{j^{*}}-X$, (a) $\bar{X}_{j^{*}-|I|}^{\prime}=\bar{X}_{j^{*}-|I|} \cup\{X-I\}$ if $j^{*}-|I| \geqslant 2$ and $(b) \underline{X}_{1}^{\prime}=\left(X-I, \underline{X}_{1}\right)$ if $\boldsymbol{j}^{*}-|\boldsymbol{I}|=\mathbf{1}$. In other words, we have $u_{j^{*}}^{\prime}=u_{j^{*}}-1$ and either (a) $u_{j^{*}-|I|}^{\prime}=u_{j^{*}-|I|}+1$ or (b) $\left|V\left(\underline{X}_{1}^{\prime}\right)\right|=\left|V\left(\underline{X}_{1}\right)\right|+1$. Observing the corresponding coefficients of $u_{j^{*}}, u_{j^{*}-|I|}$ and $\left|V\left(\underline{X}_{1}\right)\right|$ in the expression of $\alpha(j)+\beta(j)$, we have that $a_{j^{*}}-a_{j^{*}-|I|}$ and $a_{j^{*}}-b_{1}$ are at least $|I|$ by Proposition 4. Accordingly, we can conclude that $\alpha(j)+\beta(j) \geqslant \alpha^{\prime}(j)+\beta^{\prime}(j)+|I|$. (R1). Consider any subset $J \subseteq K \in \bar{X}_{k}$ with $|J|=j$ for some $k$. Notice that $j \leqslant k$. We may assume that $J \cap U \neq \emptyset$, otherwise $\sum_{w \in J} f^{\prime}(w) \geqslant F(j) \geqslant \alpha^{\prime}(j)+\beta^{\prime}(j)$ since $\alpha(j)+\beta(j) \geqslant \alpha^{\prime}(j)+\beta^{\prime}(j)+|I|$.

For the special case that $K=X-I \in \bar{X}_{j^{*}-|I|}^{\prime}, \sum_{w \in J} f^{\prime}(w)=\sum_{w \in J} f(w)$ and thus (R1) is satisfied immediately as $\alpha^{\prime}(j) \leqslant \alpha(j)$ and $\beta^{\prime}(j)=\beta(j)$.

Consider the case that $F(j)$ is saturated with respect to $J$ and $k \leqslant t$, which implies $J \nsubseteq U$ by Case 2. It follows that $\sum_{w \in J} f^{\prime}(w) \geqslant \alpha(j)+\beta(j)-(j-1)$. Since $j \leqslant k \leqslant t \leqslant|I|$, $\sum_{w \in J} f^{\prime}(w) \geqslant \alpha(j)+\beta(j)-(j-1) \geqslant \alpha^{\prime}(j)+\beta^{\prime}(j)+|I|-(j-1)>\alpha^{\prime}(j)+\beta^{\prime}(j)$.

If $F(j)$ is saturated with respect to $J$ and $k>t$, then $J \cap U$ must be empty otherwise it is either $J \subseteq U$ (Case 2) or $J \nsubseteq U$ (Case (3.3) with $u=k>t$ ), a contradiction to the assumption $J \cap U \neq \emptyset$.

If $F(j)$ is not saturated with respect to $J$, then $F(|J \cap U|)$ must not be saturated with respect to $J \cap U$ (by Case 2 ) and $|J \cap U| \leqslant t$ (by the maximality assumption of $t$ ). It follows that $\sum_{w \in J} f^{\prime}(w) \geqslant[\alpha(j)+\beta(j)+1]-|J \cap U| \geqslant \alpha^{\prime}(j)+\beta^{\prime}(j)+|I|+1-|J \cap U| \geqslant \alpha^{\prime}(j)+\beta^{\prime}(j)$ 
as $|I| \geqslant t \geqslant|J \cap U|$.

From the above discussion, in either case we have $\sum_{w \in J} f^{\prime}(w) \geqslant \alpha^{\prime}(j)+\beta^{\prime}(j)$. As $J$ is chosen arbitrarily, we can conclude that $F^{\prime}(j) \geqslant \alpha^{\prime}(j)+\beta^{\prime}(j)$ for all $j \in[m]$.

(R2). Consider $f^{\prime}(v)$ for any $v \in \underline{X}_{j, i}$.

For the special case that $v \in X-I=\underline{X}_{1,1}, f^{\prime}(v)=f(v) \geqslant F(1) \geqslant \alpha(1)+\beta(1)=$ $\sum_{p=2}^{m} u_{p}=S^{\prime}(m-1)+1$, as desired. Recall that if it is the case (a), all families remain except that $\bar{X}_{j^{*}}^{\prime}=\bar{X}_{j^{*}}-X$ and $\bar{X}_{j^{*}-|I|}^{\prime}=\bar{X}_{j^{*}-|I|} \cup\{X-I\}$. If it is the case (b), all families remain except that $\bar{X}_{j^{*}}^{\prime}=\bar{X}_{j^{*}}-X$ and $\underline{X}_{1}^{\prime}=\left(X-I, \underline{X}_{1}\right)$.

If $j \leqslant m+1-t$, then we have $v \notin V\left(\underline{X}_{j}\right) \cap U$ otherwise it is Case (3.1) with $s=m+1-j \geqslant t$. Therefore, $f^{\prime}(v)=f(v) \geqslant S(m-j)+\sum_{p=1}^{j-1}\left|V\left(\underline{X}_{p}\right)\right|+\sum_{q=1}^{i-1}\left|V\left(\underline{X}_{j, q}\right)\right|+1$. If it is the case (a), then $S^{\prime}(m-j) \leqslant S(m-j)$ (as $s_{j^{*}} \geqslant s_{j^{*}-|I|}$ by Proposition 3) and $\sum_{p=1}^{j-1}\left|V\left(\underline{X}_{p}^{\prime}\right)\right|+\sum_{q=1}^{i-1}\left|V\left(\underline{X}_{j, q}^{\prime}\right)\right|=\sum_{p=1}^{j-1}\left|V\left(\underline{X}_{p}\right)\right|+\sum_{q=1}^{i-1}\left|V\left(\underline{X}_{j, q}\right)\right|$.

If it is the case (b), then $S^{\prime}(m-j) \leqslant S(m-j)-1$ (by Proposition 3 and $u_{j^{*}}^{\prime}=u_{j^{*}}-1$ ) and $\sum_{p=1}^{j-1}\left|V\left(\underline{X}_{p}^{\prime}\right)\right|+\sum_{q=1}^{i-1}\left|V\left(\underline{X}_{j, q}^{\prime}\right)\right|=\sum_{p=1}^{j-1}\left|V\left(\underline{X}_{p}\right)\right|+\sum_{q=1}^{i-1}\left|V\left(\underline{X}_{j, q}\right)\right|+1$. In either case, $f^{\prime}(v) \geqslant S^{\prime}(m-j)+\sum_{p=1}^{j-1}\left|V\left(\underline{X}_{p}^{\prime}\right)\right|+\sum_{q=1}^{i-1}\left|V\left(\underline{X}_{j, q}^{\prime}\right)\right|+1$, as desired.

If $j>m+1-t$, then $m+1-j<j^{*}$ since $t<j^{*}$ otherwise it is Case 1 . In this case, observing the coefficients of $u_{j^{*}}$ and $u_{j^{*}-|I|}$ in $S(m-j)$, we obtain $s_{j^{*}}>s_{j^{*}-|I|}$ and $s_{j^{*}} \geqslant 2$ by Proposition 3 .

If it is the case (a), then $S(m-j) \geqslant S^{\prime}(m-j)+1$ (since $\left.s_{j^{*}}>s_{j^{*}-|I|}\right)$ and thus $f^{\prime}(v) \geqslant f(v)-1 \geqslant S^{\prime}(m-j)+\sum_{p=1}^{j-1}\left|V\left(\underline{X}_{p}^{\prime}\right)\right|+\sum_{q=1}^{i-1}\left|V\left(\underline{X}_{j, q}^{\prime}\right)\right|+1$ as $\sum_{p=1}^{j-1}\left|V\left(\underline{X}_{p}^{\prime}\right)\right|$ and $\sum_{q=1}^{i-1}\left|V\left(\underline{X}_{j, q}^{\prime}\right)\right|$ are inherited.

If it is the case (b), then $S(m-j) \geqslant S^{\prime}(m-j)+2$ (since $s_{j^{*}} \geqslant 2$ and $u_{j^{*}}^{\prime}=u_{j^{*}}-$ 1) and $\sum_{p=1}^{j-1}\left|V\left(\underline{X}_{p}^{\prime}\right)\right|+\sum_{q=1}^{i-1}\left|V\left(\underline{X}_{j, q}^{\prime}\right)\right|=\sum_{p=1}^{j-1}\left|V\left(\underline{X}_{p}\right)\right|+\sum_{q=1}^{i-1}\left|V\left(\underline{X}_{j, q}\right)\right|+1$. Thus, $f^{\prime}(v) \geqslant f(v)-1 \geqslant\left[S^{\prime}(m-j)+2\right]+\left[\sum_{p=1}^{j-1}\left|V\left(\underline{X}_{p}^{\prime}\right)\right|+\sum_{q=1}^{i-1}\left|V\left(\underline{X}_{j, q}^{\prime}\right)\right|-1\right] \geqslant S^{\prime}(m-j)+$ $\sum_{p=1}^{j-1}\left|V\left(\underline{X}_{p}^{\prime}\right)\right|+\sum_{q=1}^{i-1}\left|V\left(\underline{X}_{j, q}^{\prime}\right)\right|+1$, as desired.

Case (3.3): Note that in this case, $u$ is the largest, i.e., $u>s$ and $u>t$. To avoid confusion, instead of $u$ we shall use $j^{*}$ to denote the largest index and assume that there exists $Y \nsubseteq U$ and $Y \subseteq X \in \bar{X}_{j^{*}}$ such that $F(|Y|)$ is saturated with respect to $Y$. Among all these cases with the same $j^{*}$, we choose the one with the largest $|U \cap X|$. Let $I=U \cap X$ and $\Pi^{\prime}$ be obtained from $\Pi$ by removing $I$ where all families remain except that $\bar{X}_{j^{*}}^{\prime}=\bar{X}_{j^{*}}-X$, (a) $\overline{\boldsymbol{X}}_{j^{*}-|\boldsymbol{I}|}^{\prime}=\overline{\boldsymbol{X}}_{\boldsymbol{j}^{*}-|\boldsymbol{I}|} \cup\{\boldsymbol{X}-\boldsymbol{I}\}$ if $\boldsymbol{j}^{*}-|\boldsymbol{I}| \geqslant 2$ and (b) $\underline{X}_{1}^{\prime}=\left(\boldsymbol{X}-\boldsymbol{I}, \underline{\boldsymbol{X}}_{1}\right)$ if $\boldsymbol{j}^{*}-|\boldsymbol{I}|=1$. Notice that $u_{j^{*}}^{\prime}=u_{j^{*}}-1$ and either (a) $u_{j^{*}-|I|}^{\prime}=u_{j^{*}-|I|}+1$ or (b) $\left|V\left(\underline{X}_{1}^{\prime}\right)\right|=\left|V\left(\underline{X}_{1}\right)\right|+1$. The same argument as that in Case (3.2) implies that $\alpha(j)+\beta(j) \geqslant \alpha^{\prime}(j)+\beta^{\prime}(j)+|I|$.

(R1). Consider any subset $J \subseteq K \in \bar{X}_{k}$ with $|J|=j$ for some $k$ and $J \cap U \neq \emptyset$. Notice that $j \leqslant k$.

For the special case that $K=X-I \in \bar{X}_{j^{*}-|I|}^{\prime}$, it follows that $\sum_{w \in J} f^{\prime}(w)=\sum_{w \in J} f(w)$ and thus (R1) is satisfied immediately as $\alpha^{\prime}(j) \leqslant \alpha(j)$ and $\beta^{\prime}(j)=\beta(j)$.

If $F(j)$ is saturated with respect to $J$, then $k \leqslant j^{*}$ otherwise it is Case (3.3) with $k>j^{*}$ violating the maximality assumption of $j^{*}$. Next we discuss the two cases $k=j^{*}$ 
and $k<j^{*}$ separately.

For the case $k=j^{*}, \sum_{w \in J} f^{\prime}(w) \geqslant F(j)-|I| \geqslant \alpha(j)+\beta(j)-|I| \geqslant \alpha^{\prime}(j)+\beta^{\prime}(j)$ where the first inequality follows from the fact that among all the cases with $k=j^{*}$ we choose the one with the largest $|U \cap X|$.

If $k<j^{*}$, then $J \nsubseteq U$ (since Case 2 does not hold). It follows that $\sum_{w \in J} f^{\prime}(w) \geqslant$ $\alpha(j)+\beta(j)-(j-1)$. In addition, $j \leqslant k<j^{*}$ implies that $a_{j^{*}}-\max \left\{a_{j^{*}-|I|}, b_{1}\right\} \geqslant j-1$ by Proposition 4. It follows that $\alpha(j)+\beta(j) \geqslant \alpha^{\prime}(j)+\beta^{\prime}(j)+j-1$. Hence, $\sum_{w \in J} f^{\prime}(w) \geqslant$ $\alpha^{\prime}(j)+\beta^{\prime}(j)$.

Consider the case when $F(j)$ is not saturated with respect to $J$. It follows that $\sum_{w \in J} f^{\prime}(w) \geqslant \alpha(j)+\beta(j)+1-j$. There are two cases: $j<j^{*}$ and $j \geqslant j^{*}$.

If $j<j^{*}$, by Proposition 4 we have $a_{j^{*}}-\max \left\{a_{j^{*}-|I|}, b_{1}\right\} \geqslant j-1$. This implies that $\alpha(j)+\beta(j) \geqslant \alpha^{\prime}(j)+\beta^{\prime}(j)+j-1$ and therefore $\sum_{w \in J} f^{\prime}(w) \geqslant \alpha^{\prime}(j)+\beta^{\prime}(j)$.

If $j \geqslant j^{*}$, then we have $J \nsubseteq U$ otherwise it is Case (3.2) with $t=j \geqslant j^{*}=u$. Furthermore, it must be $|J \cap U|<j^{*}$ otherwise either $F(|J \cap U|)$ is saturated with respect to $J \cap U$ (Case 2) or $F(|J \cap U|)$ is not saturated with respect to $J \cap U$ and $t=|J \cap U| \geqslant j^{*}=u$ (Case (3.2)). Since $F(j)$ is not saturated with respect to $J$ and $|J \cap U|<j^{*}$, we have $\sum_{w \in J} f^{\prime}(w) \geqslant \alpha(j)+\beta(j)+1-\left(j^{*}-1\right)$. Since $j \geqslant j^{*}$, by Proposition 4 we have $a_{j^{*}}-\max \left\{a_{j^{*}-|I|}, b_{1}\right\} \geqslant j^{*}-2$. It follows that $\alpha(j)+\beta(j) \geqslant \alpha^{\prime}(j)+\beta^{\prime}(j)+j^{*}-2$ and thus $\sum_{w \in J} f^{\prime}(w) \geqslant \alpha^{\prime}(j)+\beta^{\prime}(j)$.

From the above discussion, in either case we have $\sum_{w \in J} f^{\prime}(w) \geqslant \alpha^{\prime}(j)+\beta^{\prime}(j)$. As $J$ is chosen arbitrarily, we can conclude that $F^{\prime}(j) \geqslant \alpha^{\prime}(j)+\beta^{\prime}(j)$ for all $j \in[m]$.

(R2). Consider $f^{\prime}(v)$ for any $v \in \underline{X}_{j, i}$.

For the special case that $v \in X-I=\underline{X}_{1,1}, f^{\prime}(v)=f(v) \geqslant F(1) \geqslant \alpha(1)+\beta(1)=$ $\sum_{p=2}^{m} u_{p}=S^{\prime}(m-1)+1$, as desired. Recall that if it is the case (a), all families remain except that $\bar{X}_{j^{*}}^{\prime}=\bar{X}_{j^{*}}-X$ and $\bar{X}_{j^{*}-|I|}^{\prime}=\bar{X}_{j^{*}-|I|} \cup\{X-I\}$. If it is the case (b), all families remain except that $\bar{X}_{j^{*}}^{\prime}=\bar{X}_{j^{*}}-X$ and $\underline{X}_{1}^{\prime}=\left(X-I, \underline{X}_{1}\right)$.

If $j \leqslant m+1-j^{*}$, then we have $v \notin V\left(\underline{X}_{j}\right) \cap U$ otherwise it is Case (3.1) with $s=m+$ $1-j \geqslant j^{*}=u$. Therefore, $f^{\prime}(v)=f(v) \geqslant S(m-j)+\sum_{p=1}^{j-1}\left|V\left(\underline{X}_{p}\right)\right|+\sum_{q=1}^{i-1}\left|V\left(\underline{X}_{j, q}\right)\right|+1$. If it is the case (a), then $S^{\prime}(m-j) \leqslant S(m-j)$ (by Proposition 3 and $j^{*} \geqslant j^{*}-|I|$ ) and $\sum_{p=1}^{j-1}\left|V\left(\underline{X}_{p}^{\prime}\right)\right|+\sum_{q=1}^{i-1}\left|V\left(\underline{X}_{j, q}^{\prime}\right)\right|=\sum_{p=1}^{j-1}\left|V\left(\underline{X}_{p}\right)\right|+\sum_{q=1}^{i-1}\left|V\left(\underline{X}_{j, q}\right)\right|$.

If it is the case (b), then $S^{\prime}(m-j) \leqslant S(m-j)-1$ (by Proposition 3 and $u_{j^{*}}^{\prime}=u_{j^{*}}-1$ ) and $\sum_{p=1}^{j-1}\left|V\left(\underline{X}_{p}^{\prime}\right)\right|+\sum_{q=1}^{i-1}\left|V\left(\underline{X}_{j, q}^{\prime}\right)\right|=\sum_{p=1}^{j-1}\left|V\left(\underline{X}_{p}\right)\right|+\sum_{q=1}^{i-1}\left|V\left(\underline{X}_{j, q}\right)\right|+1$.

In either case, $f^{\prime}(v) \geqslant S^{\prime}(m-j)+\sum_{p=1}^{j-1}\left|V\left(\underline{X}_{p}^{\prime}\right)\right|+\sum_{q=1}^{i-1}\left|V\left(\underline{X}_{j, q}^{\prime}\right)\right|+1$ as desired.

If $j>m+1-j^{*}$, then $j^{*}>m+1-j$. If it is the case (a), then to verify $f^{\prime}(v)$ it suffices to compare the coefficients of $u_{j^{*}}$ and $u_{j^{*}-|I|}$ in $S(m-j)$. Since $j^{*}>m+1-j$, we have $s_{j^{*}}>s_{j^{*}-|I|}$ by Proposition 3. Consequently, $S^{\prime}(m-j) \leqslant S(m-j)-1$. Thus, $f^{\prime}(v) \geqslant S^{\prime}(m-j)+\sum_{p=1}^{j-1}\left|V\left(\underline{X}_{p}^{\prime}\right)\right|+\sum_{q=1}^{i-1}\left|V\left(\underline{X}_{j, q}^{\prime}\right)\right|+1$.

Otherwise, if it is the case (b), then $\sum_{p=1}^{j-1}\left|V\left(\underline{X}_{p}^{\prime}\right)\right|+\sum_{q=1}^{i-1}\left|V\left(\underline{X}_{j, q}^{\prime}\right)\right|=\sum_{p=1}^{j-1}\left|V\left(\underline{X}_{p}\right)\right|+$ $\sum_{q=1}^{i-1}\left|V\left(\underline{X}_{j, q}\right)\right|+1$. Since $j^{*}>m+1-j$, the term $u_{j^{*}}$ in $S(m-j)$ has a coefficient $s_{j^{*}} \geqslant 2$ by Proposition 3. It follows that $S(m-j) \geqslant S^{\prime}(m-j)+2$ as $u_{j^{*}}^{\prime}=u_{j^{*}}-1$. Thus, $f^{\prime}(v) \geqslant f(v)-1 \geqslant S(m-j)+\sum_{p=1}^{j-1}\left|V\left(\underline{X}_{p}\right)\right|+\sum_{q=1}^{i-1}\left|V\left(\underline{X}_{j, q}\right)\right| \geqslant\left(S^{\prime}(m-j)+2\right)+$ 
$\left(\sum_{p=1}^{j-1}\left|V\left(\underline{X}_{p}^{\prime}\right)\right|+\sum_{q=1}^{i-1}\left|V\left(\underline{X}_{j, q}^{\prime}\right)\right|-1\right) \geqslant S^{\prime}(m-j)+\sum_{p=1}^{j-1}\left|V\left(\underline{X}_{p}^{\prime}\right)\right|+\sum_{q=1}^{i-1}\left|V\left(\underline{X}_{j, q}^{\prime}\right)\right|+1$.

As one of the above cases must occur, by induction the proof is complete.

\section{Consequences of Theorem 6}

Theorem 6 provides a sufficient condition on $f$ for graphs being on-line $f$-choosable. It is a widely applicable tool for computing the on-line choice number of complete multipartite graphs with varying parameters. Many interesting results can be obtained immediately from Theorem 6. This section provides only some of them that are relevant to recent results.

Theorem 7. Let $G$ be a complete multipartite graph with independence number $m \geqslant$ 2 and let $k_{p}$ denote the number of parts of cardinality $p$ for $1 \leqslant p \leqslant m$. If $k_{1}-$ $\sum_{p=2}^{m}\left(\frac{p^{2}}{2}-\frac{3 p}{2}+1\right) k_{p} \geqslant 0$, then $G$ is on-line chromatic-choosable.

Proof. Consider the particular partition $\Pi=\left\{\underline{X}_{m-1}, \underline{X}_{m-2}, \ldots, \underline{X}_{1}, \bar{X}_{2}, \ldots, \bar{X}_{m}\right\}$ of parts of $G$ where $\underline{X}_{m-1}, \underline{X}_{m-2}, \ldots, \underline{X}_{2}$ are empty sets. Note that when all the families $\underline{X}_{m-1}, \underline{X}_{m-2}, \ldots, \underline{X}_{2}$ are empty, the remaining families are determined exactly. That is $\ell_{m-1}=\ell_{m-2}=\cdots=\ell_{2}=0, \ell_{1}=k_{1}$ and $u_{p}=k_{p}$ for $p=2,3, \ldots, m$. Obviously, $\sum_{p=2}^{m} u_{p}+\ell_{1}=\sum_{p=1}^{m} k_{p}=\chi(G)$. Let $f(v)=\chi(G)$ for all $v \in V(G)$.

Next, we will verify that $f$ and $\Pi$ with arbitrarily ordered $\underline{X}_{1}$ satisfy (R1) and (R2) in Theorem 6. The inequality (R2) holds as $f(v)=\sum_{p=2}^{m} u_{p}+\ell_{1} \geqslant S(m-1)+$ $\sum_{q=1}^{i-1}\left|V\left(\underline{X}_{1, q}\right)\right|+1$ for all $v \in V\left(\underline{X}_{1, i}\right)$.

Consider any subset $J \subseteq X \in \bigcup_{p=2}^{m} \bar{X}_{p}$ with $|J|=j, 1 \leqslant j \leqslant m$. To verify (R1), it suffices to prove that $\sum_{w \in J} f(w) \geqslant \alpha(j)+\beta(j)$, or equivalently that

$$
\begin{aligned}
& j\left(\sum_{p=2}^{m} u_{p}+\ell_{1}\right) \geqslant \sum_{p=2}^{j}\left(j+\frac{p^{2}}{2}-\frac{3 p}{2}+1\right) u_{p} \\
&+\sum_{p=j+1}^{m}\left(\frac{j}{2}-\frac{j^{2}}{2}+p j-p+1\right) u_{p}+(j-1) \ell_{1} \\
& \Leftrightarrow \quad \ell_{1}-\sum_{p=2}^{j}\left(\frac{p^{2}}{2}-\frac{3 p}{2}+1\right) u_{p}-\sum_{p=j+1}^{m}\left(-\frac{j}{2}-\frac{j^{2}}{2}+p j-p+1\right) u_{p} \geqslant 0 .
\end{aligned}
$$

Obviously, Eq.(4) is always true for $j=1$. By using elementary calculus, it is easy to prove that for all $j=2,3, \ldots, m$ with $j \leqslant p-1,\left(\frac{p^{2}}{2}-\frac{3 p}{2}+1\right)-\left(-\frac{j}{2}-\frac{j^{2}}{2}+p j-p+1\right) \geqslant 0$. Thus, to prove Eq.(4) for all $j \in[m]$, it suffices to show that

$$
\ell_{1}-\sum_{p=2}^{m}\left(\frac{p^{2}}{2}-\frac{3 p}{2}+1\right) u_{p} \geqslant 0 .
$$


This is trivially true as $k_{1}-\sum_{p=2}^{m}\left(\frac{p^{2}}{2}-\frac{3 p}{2}+1\right) k_{p} \geqslant 0$. By Theorem $6, G$ is on-line chromatic-choosable.

For any two graphs $G$ and $H$, denote by $G+H$ the join of $G$ and $H$, that is, the disjoint union of $G$ and $H$ with the edges $\{u v: u \in V(G), v \in V(H)\}$. Kozik, Micek and Zhu [10] proved that for any graph $G$, the join of $G$ and a complete graph of order $|V(G)|^{2}$ is on-line chromatic-choosable. Later, Carraher, Loeb, Mahoney, Puleo, Tsai and West [2] improved upon $|V(G)|^{2}$ with an additional assumption. Precisely, they proved that for every $d$-degenerate graph $G$ having an optimal proper coloring with color classes of size at most $m, G+K_{t}$ is on-line chromatic-choosable if $t \geqslant(m+1) d$. Theorem 7 also provides an alternative result in this aspect.

Corollary 8. Let $G$ be a graph of independence number at most $m$ and have an optimal proper coloring where there are $k_{i}$ color classes of cardinality $i$. If

$$
t \geqslant \sum_{p=2}^{m}\left(\frac{p^{2}}{2}-\frac{3 p}{2}+1\right) k_{p}-k_{1}
$$

then $G+K_{t}$ is on-line chromatic-choosable.

Kozik, Micek and Zhu [10] commented that when $|V(G)| \leqslant \chi(G)+\sqrt{\chi(G)}$, then $G$ is on-line chromatic-choosable. Later, Carraher et al. [2] showed that the same conclusion holds under a relaxed condition $|V(G)| \leqslant \chi(G)+2 \sqrt{\chi(G)-1}$. They proposed a weak version of Conjecture 2:

Conjecture 9 (Weak On-Line Ohba's Conjecture). [2] There is a constant $c \in(1,2]$ such that $\chi_{p}(G)=\chi(G)$ whenever $|V(G)| \leqslant c \chi(G)$.

The weak on-line Ohba's conjecture is still open, to the best of our knowledge. Following the same argument in Theorem 7, we obtain the following result, which goes one further step towards the weak conjecture.

Corollary 10. If $G$ is a graph with independence number $m \geqslant 2$ and

$$
|V(G)| \leqslant \frac{m^{2}-m+2}{m^{2}-3 m+4} \chi(G)
$$

then $\chi(G)=\chi_{p}(G)$.

Proof. As $|V(G)| \leqslant \frac{m^{2}-m+2}{m^{2}-3 m+4} \chi(G), \chi(G)=\ell_{1}+\sum_{p=2}^{m} u_{p}$, and $|V(G)|=\ell_{1}+\sum_{p=2}^{m} p u_{p}$, we have

$$
\ell_{1} \geqslant \sum_{p=2}^{m}\left[\frac{p\left(m^{2}-3 m+4\right)-\left(m^{2}-m+2\right)}{2 m-2}\right] u_{p} .
$$

According to Eq.(5), it suffices to show that when $2 \leqslant p \leqslant m$,

$$
\frac{p\left(m^{2}-3 m+4\right)-\left(m^{2}-m+2\right)}{2 m-2}-\left(\frac{p^{2}}{2}-\frac{3 p}{2}+1\right) \geqslant 0 .
$$


Simplifying yields $(m-1) p^{2}-\left(m^{2}+1\right) p+m^{2}+m \leqslant 0$. Consider the quadratic function $g(p)=(m-1) p^{2}-\left(m^{2}+1\right) p+m^{2}+m$. Obviously, $g(2) \leqslant 0$ and $g(m) \leqslant 0$ whenever $m \geqslant 2$. Thus, one can conclude that $g(p) \leqslant 0$ whenever $2 \leqslant p \leqslant m$. This completes the proof.

Consequently, any graph $G$ with $|V(G)| \leqslant 2 \chi(G)$ and independence number $m \leqslant 3$ is on-line chromatic-choosable; the same conclusion was proved independently in $[6,8]$ for $m=2$ and in [10] for $m \leqslant 3$.

Alon [1] established the asymptotically tight bound $\chi_{\ell}\left(K_{m \star k}\right)=\Theta(k \log m)$. The following result, which is another immediate consequence of Theorem 6 , gives a general upper bound for $\chi_{p}\left(K_{m \star k}\right)$.

Corollary 11. For any integer $m \geqslant 3, \chi_{p}\left(K_{m \star k}\right) \leqslant\left(m+\frac{1}{2}-\sqrt{2 m-2}\right) k$.

Proof. Consider the partition $\Pi=\left\{\underline{\underline{X}}_{m-1}, \underline{X}_{m-2}, \ldots, \underline{X}_{1}, \bar{X}_{2}, \ldots, \bar{X}_{m}\right\}$ of parts of $K_{m \star k}$ where all families are empty except $\bar{X}_{m}$, i.e., $u_{m}=k$ and $\ell_{1}=\cdots=\ell_{m-1}=u_{2}=\cdots=$ $u_{m-1}=0$. Let $f(v)=\left(m+\frac{1}{2}-\sqrt{2 m-2}\right) k$ for all $v \in V\left(K_{m \star k}\right)$. The corollary follows directly from Theorem 6 with the specified $(\Pi, f)$.

Particularly, when $m=3$, it assures the same conclusion in [10] that $\chi_{p}\left(K_{3 \star k}\right) \leqslant \frac{3}{2} k$.

\section{Acknowledgements}

The authors would like to thank the anonymous referees for their valuable suggestions.

\section{References}

[1] N. Alon. Choice numbers of graphs: a probabilistic approach. Combin. Probab. Comput., 1(02):107-114, 1992.

[2] J. Carraher, S. Loeb, T. Mahoney, G. Puleo, M. Tsai, and D. West. Extending graph choosability results to paintability. Manuscript, 2011.

[3] P. Erds, A. L. Rubin, and H. Taylor. Choosability in graphs. Congr. Numer., 26:125157, 1979.

[4] F. Galvin. The list chromatic index of a bipartite multigraph. J. Combin. Theory B, 63(1):153-158, 1995.

[5] S. Gravier. A Hajs-like theorem for list coloring. Discrete Math., 152(1):299-302, 1996.

[6] P.-Y. Huang, T.-L. Wong, and X. Zhu. Application of polynomial method to on-line list colouring of graphs. Eur. J. Combin., 33(5):872-883, 2012.

[7] T. R. Jensen and B. Toft. Graph coloring problems. Wiley-Interscience Series in Discrete Mathematics and Optimization. John Wiley \& Sons, New York, 1995.

[8] S.-J. Kim, Y. S. Kwon, D. D.-F. Liu, and X. Zhu. On-line list colouring of complete multipartite graphs. Electron. J. Combin., 19(1):P41, 2012. 
[9] A. V. Kostochka and D. R. Woodall. Choosability conjectures and multicircuits. Discrete Math., 240(1):123-143, 2001.

[10] J. Kozik, P. Micek, and X. Zhu. Towards an on-line version of Ohbas conjecture. Eur. J. Combin., 36:110-121, 2014.

[11] J. A. Noel, B. A. Reed, and H. Wu. A proof of a conjecture of Ohba. J. Graph Theory, doi:10.1002/jgt.21819, 2014.

[12] K. Ohba. On chromatic-choosable graphs. J. Graph Theory, 40(2):130-135, 2002.

[13] U. Schauz. Mr. Paint and Mrs. Correct. Electron. J. Combin., 16(1):R77, 2009.

[14] V. G. Vizing. Coloring the vertices of a graph in prescribed colors. Diskret. Analiz., 29(3):10, 1976.

[15] X. Zhu. On-line list colouring of graphs. Electron. J. Combin., 16(1):R127, 2009. 